\title{
A Long-term Follow-up of Serum Myeloperoxidase Antineutrophil Cytoplasmic Antibodies (MPO-ANCA) in Patients with Graves Disease Treated with Propylthiouracil
}

\author{
REIKo ISHII ${ }^{1)}$, Misa IMAIZUMI ${ }^{1,2)}$, AKANE IDE ${ }^{1)}$, NobUKo SERA ${ }^{2)}$, IKUKo UEKI'), ICHIRO HORIE ${ }^{1)}$, \\ TAKAO ANDO ${ }^{1)}$, TOSHIRO USA ${ }^{1)}$, ERI EJIMA ${ }^{3)}$, KIYOTO ASHIZAWA ${ }^{4)}$ AND KATSUMI EGUCHI ${ }^{1)}$ \\ ${ }^{1)}$ Department of Endocrinology and Metabolism, Unit of Translational Medicine, Graduate School of Biomedical Sciences, \\ Nagasaki University, Nagasaki, Japan \\ ${ }^{2)}$ Department of Clinical Studies, Radiation Effects Research Foundation, Nagasaki, Japan \\ ${ }^{3)}$ Department of Internal Medicine, National Hospital Organization Saga National Hospital, Saga, Japan \\ ${ }^{4)}$ Department of Internal Medicine, Saiseikai Nagasaki Hospital, Nagasaki, Japan
}

\begin{abstract}
Propylthiouracil (PTU) is known to induce myeloperoxidase antineutrophil cytoplasmic antibodies (MPOANCA) in patients with Graves disease (GD). Previously, we showed that serum MPO-ANCA were frequently seen in patients with GD treated with PTU. In this study, we analyzed 13 patients with positive MPO-ANCA examining a longterm clinical consequence of these patients as well as antibody titers during $5.6 \pm 3.0$ years. PTU therapy was continued in 8 patients and discontinued in 5 patients. Antibody titers decreased in 7 of 8 patients who discontinued PTU therapy but remained positive in 5 patients 5 years after PTU withdrawal. The initial MPO-ANCA levels were significantly higher in those antibody titers remained positive for longer than 5 years $(n=5)$ than in those titers turned to be negative within 5 years after PTU withdrawal ( $\mathrm{n}=3)(203 \pm 256 \mathrm{EU}$ and $22 \pm 2 \mathrm{EU}$, respectively, $P=0.04)$, but there were no significant differences in age, gender, duration of PTU therapy or dosage of PTU. Among 5 patients who continued PTU therapy, 2 patients with initially low MPO-ANCA titers turned to having negative antibody. No patients had new symptoms or signs of vasculitis throughout the follow-up periods. The long-term follow-up study suggests that higher MPO-ANCA levels remain positive for years after PTU withdrawal but are rarely associated with vasculitis.
\end{abstract}

Key words: MPO-ANCA, Propylthiouracil, Graves disease

ANTINEUTROPHIL cytoplasmic antibodies (ANCA) have been well recognized as an important serological marker of systemic vasculitis including Wegener granulomatosis, microscopic polyarteritis, and Churg-Strauss syndrome. ANCA are also known to be associated with drug-induced vasculitis, hydralazine and antithyroid drugs being most commonly implicated [1]. The first case with ANCApositive hypersensitivity vasculitis was reported by Stankus and Johnson in 1992 in a patient with

Received Aug. 7, 2009; Accepted Oct. 15, 2009 as K09E-230 Released online in J-STAGE as advance publication Oct. 23, 2009

Correspondence to: Toshiro USA, M.D., Department of Endocrinology and Metabolism, Nagasaki University Hospital, 1-7-1 Sakamoto, Nagasaki, 852-8501, Japan.

E-mail: t-usa@nagasaki-u.ac.jp
Graves disease (GD) who was under treatment with propylthiouracil (PTU) [2]. Thereafter, a lot of similar cases complicated with ANCA-related vasculitis have been reported in GD patients mostly taking PTU [3], and the prevalence of ANCA among those under PTU therapy has been shown ranging from 4.1 to $64 \%$ [4-8]. It has been also shown that ANCA induced by PTU are almost exclusively against myeloperoxidase (MPO), but not proteinase-3 [9].

It has been known that clinical pictures associated with ANCA seen in a patient with GD treated with PTU are markedly different from those with idiopathic systemic vasculits. This is because most GD patients with positive MPO-ANCA induced by PTU are asymptomatic $[8,10,11]$. However, it has been reported that a few patients indeed develop systemic 
Table 1. Clinical backgrounds of patients with Graves disease who discontinued PTU therapy

\begin{tabular}{cccccccccc}
\hline $\begin{array}{l}\text { Patient } \\
\text { No. }\end{array}$ & $\begin{array}{c}\text { Age } \\
\text { (years) }\end{array}$ & Gender & $\begin{array}{c}\text { Duration } \\
\text { of PTU } \\
\text { therapy } \\
\text { (years) }\end{array}$ & $\begin{array}{c}\text { Average daily } \\
\text { dosage of } \\
\text { PTU (mg/day) }\end{array}$ & $\begin{array}{c}\text { MPO-ANCA } \\
\text { at the initial } \\
\text { measurement (EU) }\end{array}$ & $\begin{array}{c}\text { MPO-ANCA } \\
\text { at the end of } \\
\text { follow-up } \\
\text { period (EU) }\end{array}$ & $\begin{array}{c}\text { Follow- } \\
\text { up period } \\
\text { (years) }\end{array}$ & $\begin{array}{c}\text { Duration } \\
\text { after PTU } \\
\text { withdrawal } \\
\text { (years) }\end{array}$ & $\begin{array}{c}\text { Therapy } \\
\text { after PTU } \\
\text { withdrawal }\end{array}$ \\
\hline 1 & 25 & F & 10.0 & 152 & $21(0.2)^{*}$ & $<10$ & 4.9 & 5.1 & None \\
2 & 51 & M & 15.0 & 211 & $21(0.5)^{*}$ & $<10$ & 4.3 & 4.8 & None \\
3 & 33 & F & 3.3 & 139 & 25 & $<10$ & 3.6 & 3.6 & MMI \\
4 & 46 & F & 10.1 & 70 & $52(2.8)^{*}$ & $<10$ & 5.9 & 8.8 & RI \\
5 & 33 & F & 8.5 & 319 & $62(2.5)^{*}$ & 50 & 3.3 & 5.8 & None \\
6 & 33 & F & 9.6 & 121 & 90 & 34 & 6.2 & 6.2 & None \\
7 & 72 & F & 11.5 & 119 & 157 & 21 & 7.6 & 7.6 & MMI \\
8 & 40 & F & 2.5 & 145 & 655 & 174 & 10.2 & 10.2 & RI \\
\hline
\end{tabular}

* indicates years after PTU withdrawal in the indicated patient as shown within the parentheses since PTU was discontinued at the initial measurement of MPO-ANCA. RI indicates radioiodine therapy.

vasculitis syndromes [12], although clinical findings are less severer than those seen in patients with idiopathic systemic vasculitis [9]. It has been recognized that the initial clinical manifestations most frequently seen in vasculitis induced by antithyroid drugs are arthralgia and myalgia as well as skin manifestations, especially urticaria-like vasculitis [9]. A few severe cases with PTU-induced vasculitis have also been reported [13]. Therefore, in order to avoid the adverse consequences, it should be important to know the long term consequence of patients with GD with positive MPO-ANCA but without clinical symptoms or signs derived from vasculitis.

We have previously reported that serum MPOANCA were frequently $(37.5 \%)$ seen in patients with GD treated with PTU and the risk of MPOANCA production is increased with prolongation of PTU therapy [4]. In this study, we examined a longterm clinical consequence, including antibody titers of MPO-ANCA, of these patients positive for MPOANCA after PTU withdrawal or while PTU therapy was continued.

\section{Materials and Methods}

In the previous study [4], MPO-ANCA were detected in 21 out of 56 Japanese patients with GD treated with PTU in our department between 1996 and 1999. Among 21 ANCA-positive patients, 13 (mean age: 42 years, 2 men and 11 women) were retrospectively analyzed in this study because serum levels of MPO-ANCA determined more than one year after the initial detection were available in these patients. The mean duration between the initial and last measurements of MPO-ANCA was 5.6 \pm 3.0 years (mean $\pm \mathrm{SD}$, range 1.3-10.1 years). Written informed consent was obtained from all participants. Of 13, eight patients discontinued PTU therapy; four and four patients discontinued before and after the initial measurements of MPO-ANCA, respectively. Five patients continued PTU therapy during the followup periods. The decision whether to continue or discontinue PTU was made in an each patient after disclosing the results of MPO-ANCA and the potential risks of continuing PTU therapy as well as discussing with the patient.

We measured serum MPO-ANCA using an enzyme-linked immunosorbent assay (NIPRO, Osaka, Japan) referred as SRL kit in the previous study [4]. Because the commercial assay was modified in 2002, MPO-ANCA values measured before 2002 were converted according to the manufacturer's instruction $(y=1.106 x+6.226)$. The cut-off value was set at 20 EU according to the manufacturer's recommendation. The intervals of MPO-ANCA measurement were left to the discretion of the treating physicians. Urinary examination was conducted at least every 6 months and every time at the MPO-ANCA measurement.

Statistical analyses were performed by Wilcoxon rank sum test and Fisher exact test. All significance tests were two-sided and $P$ values of less than 0.05 were considered significant.

\section{Results}

\section{Patients who discontinued PTU therapy}

The clinical backgrounds of 8 patients who discontinued PTU therapy are summarized in Table 1 (mean follow-up period of 5.7 years). The initial 


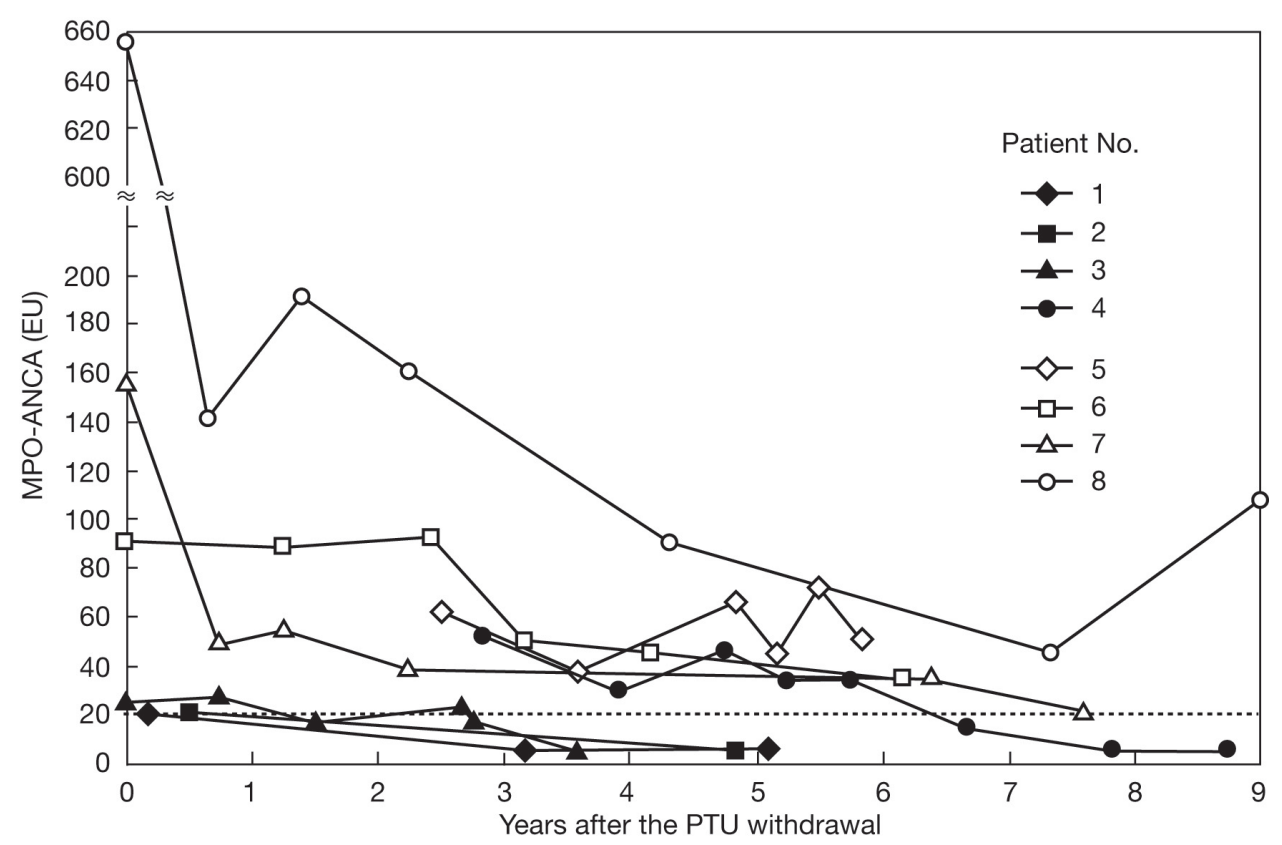

Fig. 1. MPO-ANCA in patients with Graves disease who discontinued PTU therapy.

The horizontal axis shows years after the each patient discontinued PTU therapy and the vertical axis shows antibody titers of MPO-ANCA. Antibody titers higher than $20 \mathrm{EU}$, as indicated by a dash line, were considered positive. It should be noted that the initial data were obtained only after a few months to years after PTU withdrawal in some patients.

Table 2. Clinical parameters of group 1 (patients with MPO-ANCA initially positive turning to be negative within 5 years) and group 2 (patients with MPO-ANCA remained positive for more than 5 years)

\begin{tabular}{lccc}
\hline & Group 1 $(\mathrm{n}=3)$ & Group 2 $(\mathrm{n}=5)$ & $P$ \\
\hline Age & $36 \pm 13$ & $45 \pm 16$ & 0.54 \\
Gender (M:F) & $1: 2$ & $0: 5$ & 0.38 \\
Duration of PTU thrapy (years, mean \pm SD) & $9.4 \pm 5.9$ & $8.4 \pm 3.5$ & 0.77 \\
Average daily dosage of PTU (mg/day) & $167 \pm 38$ & $155 \pm 96$ & 0.37 \\
Initial MPO-ANCA (EU, mean \pm SD) & $22 \pm 2$ & $203 \pm 256$ & 0.04 \\
(range) & $21-25$ & $52-655$ & \\
\hline
\end{tabular}

Group 1 consists of 3 patients (Patient \# 1,2, and 3) whose MPO-ANCA turned to be negative within 5 years after PTU withdrawal and group 2 consists of 5 patients (Patient \# 4, 5, 6, 7 and 8) whose MPO-ANCA remained positive for more than 5 years.

serum levels of MPO-ANCA widely distributed from 21 to 655 EU. During the observation period, serum levels of MPO-ANCA decreased in all patients except Patient \# 5 (Figure 1). MPO-ANCA in 3 patients (Patient \# 1,2, and 3) turned to be negative within 5 years after PTU withdrawal (group 1), while remained positive for more than 5 years in 5 patients (Patient \# 4, 5, 6, 7 and 8, group 2). We noticed that there was no overlap in MPO-ANCA titers between group 1 and group 2, and the initial MPO-ANCA levels were significantly higher in group $2(203 \pm 256$ $\mathrm{EU})$ than in group $1(22 \pm 2 \mathrm{EU})(P=0.04)$. Other pa- rameters including age, sex, duration of PTU therapy and average daily dosage of PTU were not significantly different between two groups.

During observation periods, clinical symptoms which seemed to be related to vasculitis were only seen in one patient. In Patient \# 8, arthralgia and purpura were observed during PTU therapy [4], and these symptoms improved and disappeared after withdrawal. We also observed a continuous common cold like symptom seen in Patient \#6 as previously reported [4], but this disappeared before terminating PTU therapy, therefore, we did not consider this to be associated with 
Table 3. Clinical backgrounds of patients who continued PTU therapy

\begin{tabular}{ccccccc}
\hline $\begin{array}{l}\text { Patient } \\
\text { No. }\end{array}$ & $\begin{array}{c}\text { Age } \\
\text { (years) }\end{array}$ & Gender & $\begin{array}{c}\text { Duration of PTU } \\
\text { therapy* } \\
\text { (years) }\end{array}$ & $\begin{array}{c}\text { MPO-ANCA } \\
\text { at the initial } \\
\text { measurements (EU) }\end{array}$ & $\begin{array}{c}\text { MPO-ANCA at the } \\
\text { end of follow-up } \\
\text { period (EU) }\end{array}$ & $\begin{array}{c}\text { Follow-up } \\
\text { period (years) }\end{array}$ \\
\hline 9 & 37 & F & 5.8 & 23 & 17 & 10.2 \\
10 & 26 & M & 0.9 & 23 & 44 & 1.3 \\
11 & 62 & F & 10.2 & 24 & 18 & 4.0 \\
12 & 54 & F & 10.0 & 31 & 28 & 2.2 \\
13 & 33 & F & 5.0 & 59 & 63 & $9.6^{* *}$ \\
\hline
\end{tabular}

* Duration of PTU therapy at the initial measurement of MPO-ANCA.

** PTU therapy was discontinued 8.4 years after the initial measurement of MPO-ANCA.

PTU therapy. No other patients complained purpura or newly developed other symptoms or signs, including abnormal findings in the urinalysis, suggesting vasculitis after withdrawal of PTU.

\section{Patients who continued PTU therapy}

The clinical backgrounds of 5 patients who continued PTU therapy are summarized in Table 3 (mean follow-up period of 5.5 years). The initial MPO-ANCA levels in these patients were relatively low, ranging from 23 to $59 \mathrm{EU}$.

MPO-ANCA remained positive in three patients (Patient \# 10, 12, and 13). PTU therapy for Patient \#10 was discontinued after 1.5 years because the MPO-ANCA level increased from $23 \mathrm{EU}$ to $44 \mathrm{EU}$ and he was euthyroid with small dosage of PTU. Patient \#12 dropped out of the follow-up after 2.2 years because she stopped coming to our hospital. PTU therapy for Patient \#13 was discontinued after the initial measurement of MPO-ANCA because of the relatively high level of MPO-ANCA (59EU), and methimazole (MMI) therapy was started after giving a detail explanation of possible complications relating vasculitis. After 10 days, she complained continuous watery diarrhea and stopped taking MMI. She refused to undergo MMI therapy, radioiodine therapy and surgery and hoped to be treated with PTU. PTU therapy was restarted and she did not agree any other therapies in spite of physician's advices throughout the follow-up period. She spontaneously became hypothyroidism and PTU therapy was discontinued after 8.4 years.

On the other hand, MPO-ANCA turned to be negative in two patients (Patient \# 9 and 11, Figure 2) whose initial MPO-ANCA level was low (23EU and 24EU, respectively). Of note, therapeutic doses of PTU were increased in both Patient \# 9 (from $50 \mathrm{mg}$ every three days to $150 \mathrm{mg}$ /day) and Patient \# 11 (from 50 to $100 \mathrm{mg}$ /day) during the follow-up periods.

Three patients (Patient \# 11, 12 and 13) had a continuous common cold like symptom or myalgia at the beginning of the observation as previously reported [4], but the symptoms spontaneously disappeared. No other patients complained purpura or developed any other symptoms or signs suggesting vasculitis throughout the follow-up periods, including urinalysis studies.

\section{Discussion}

In the present study, we showed that MPO-ANCA induced by PTU decreased their titers after PTU withdrawal in most GD patients. We also showed that MPO-ANCA remain positive for longer than five years if the initial titers were high, however, which was not necessarily associated with a development of vasculitis in patients discontinued PTU therapy. In addition, antibodies with low titers disappeared within a few years when PTU was terminated, and this was also observed without discontinuing PTU in some patients.

It has been shown that, in the prospective studies, MPO-ANCA were induced after PTU therapy in patients with GD who had been originally negative for MPO-ANCA [7, 11]. ANCA have been shown to be induced by PTU in patients with GD as well as those with toxic multinodular goiter with similar frequencies [10]. We have shown in this study that serum levels of MPO-ANCA were decreased in most patients with GD after withdrawal of PTU over five years of our observation, which is in agreement with the previous observations up to 2 years $[10,11,14]$. On the other hand, other antithyroid drugs such as MMI and carbimazole have rarely been associated with MPOANCA $[4,5,8,15]$. Taken together, PTU therapy 


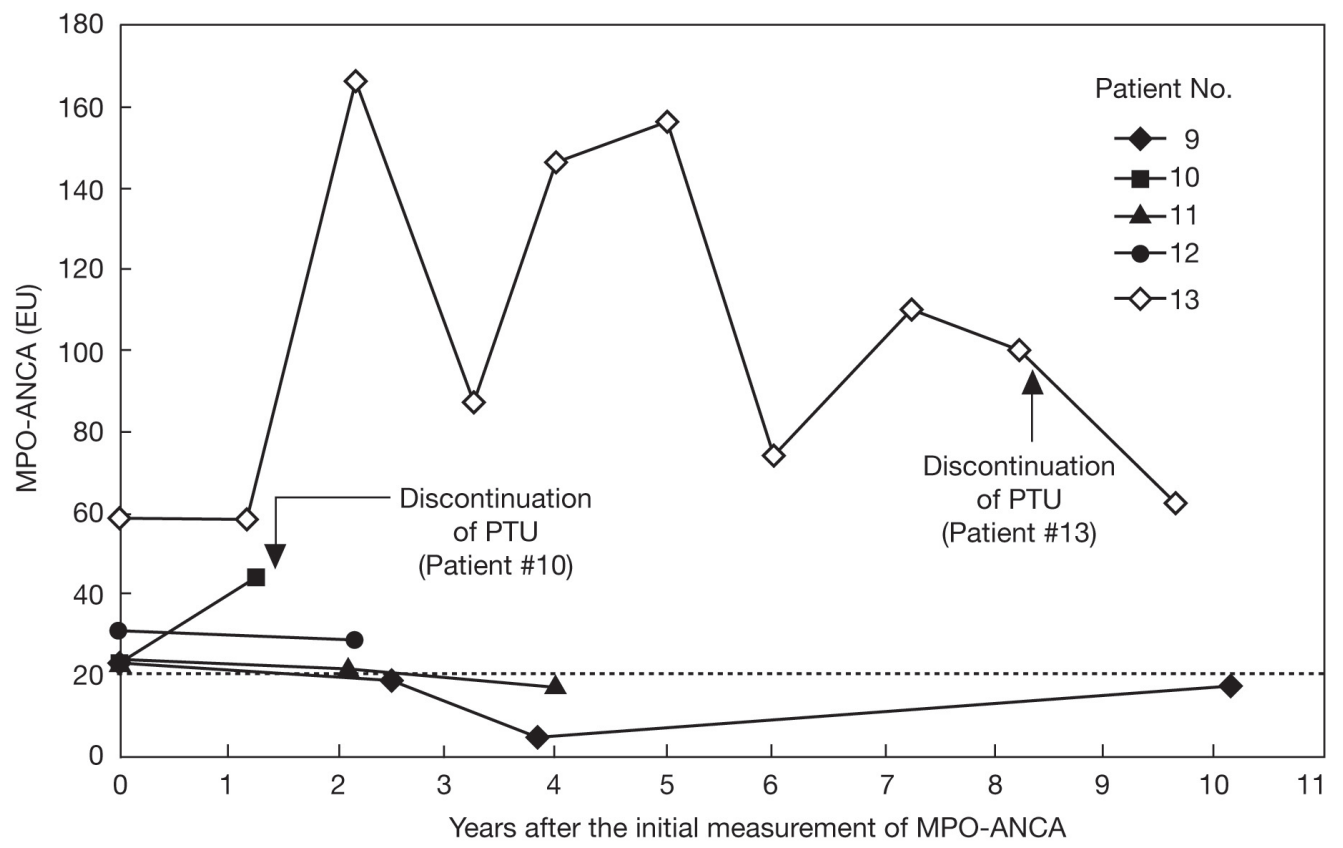

Fig. 2. MPO-ANCA in patients with Graves disease who continued PTU therapy

The horizontal axis shows years after the initial detection of MPO-ANCA in the each patient and the vertical axis shows antibody titers of MPO-ANCA. Antibody titers higher than $20 \mathrm{EU}$, as indicated by a dash line, were considered positive. PTU therapy was discontinued in Patient \#10 and 13 during the follow-up periods 1.5 and 8.4 years after the initial measurements of MPO-ANCA, respectively.

rather than GD itself might be closely associated with MPO-ANCA. Although the precise mechanism(s) how MPO-ANCA develops remain unclear, PTU has been shown to accumulate in the neutrophils [16] and to bind the MPO antigen with altering its structure [17]. It has been, therefore, suggested that the structural change of MPO antigen induced might promote development of ANCA in susceptible individuals [18].

Our study showed that higher serum levels of MPOANCA remained positive for more than 5 years after PTU withdrawal. It was observed that serum levels of MPO-ANCA decreased without immunosuppressive therapy following terminating PTU or more importantly developing vasculitis. It has recently been shown that higher titers of MPO-ANCA induced by PTU were associated with clinical vasculitis [19]. Our findings may, thus, suggest that discontinuation of PTU therapy seems to decrease the potential risks for the adverse consequences in those with high MPOANCA titers. Patients should be carefully observed to find early clinical signs and symptoms relating vasculitis even after PTU withdrawal, but frequent MPO-ANCA measurements (e.g. more than every 6 months) may not be necessary.
In contrast, we also observed that, when serum levels of MPO-ANCA were low, antibody titers decreased and eventually became negative after PTU withdrawal. This was also the case when PTU was continued in some patients with GD, without inducing vasculitis. There has been no randomized controlled trial whether to continue or discontinue PTU therapy in such patients and we cannot deny a possible bias for the decision by the physician in this study. Therefore, it is difficult to make a conclusion whether to continue or discontinue PTU therapy in patients with low serum levels of MPO-ANCA. A recent study also suggests no association between serum levels of MPO-ANCA and severity of vasculitis in MPO-ANCA associated vasculitis by antithyroid drugs [20]. Although previous studies strongly support a primary pathogenic role of MPO-ANCA for inducing vasculitis, the pathogenesis of ANCA-related disease is multifactorial and is influenced by genetic susceptibility and environmental factors such as infections [21]. It is suggested that patients known to have low levels of MPO-ANCA should be under a close observation in order to find early clinical signs and symptoms relating vasculitis in addition to measure MPO-ANCA periodically when 
PTU therapy is continued. PTU withdrawal should be considered to avoid adverse consequence when serum levels of MPO-ANCA increase. Detail explanations to patients and their agreements are necessary to continue PTU therapy.

In summary, the present long-term follow-up study demonstrated that high levels of MPO-ANCA induced by PTU persisted for years after PTU withdrawal although it might not necessarily be associated with development of vasculitis. Low levels of MPOANCA became negative sooner after PTU withdrawal and turned to be negative in some patients without discontinuing PTU. However, we should not forget a possible risk of vasculitis even in patients with low MPO-ANCA levels when PTU therapy is continued. A further study with more patients is necessary to clarify a long-term clinical significance of PTUinduced MPO-ANCA and to provide clinical insights into the managements of such patients.

\section{Acknowledgments}

We thank Ms. Masako Ishikawa for her secretarial assistance.

\section{References}

1. Hoffman, G.S. \& Specks, U. (1998) Antineutrophil cytoplasmic antibodies. Arthritis Rheum 41, 1521-1537.

2. Stankus, S.J. \& Johnson, N.T. (1992) Propylthiouracilinduced hypersensitivity vasculitis presenting as respiratory failure. Chest 102, 1595-1596.

3. Ashizawa, K. \& Eguchi, K. (2003) Serum anti-myeloperoxidase antineutrophil cytoplasmic antibodies (MPO-ANCA) in patients with Graves' disease receiving anti-thyroid medication. Intern Med 42, 463-464.

4. Sera, N., Ashizawa, K., Ando, T., Abe, Y., Ide, A., Usa, T., Tominaga, T., Ejima, E., Yokoyama, N. \& Eguchi, K. (2000) Treatment with propylthiouracil is associated with appearance of antineutrophil cytoplasmic antibodies in some patients with Graves' disease. Thyroid 10, 595-599.

5. Sato, H., Hattori, M., Fujieda, M., Sugihara, S., Inomata, H., Hoshi, M. \& Miyamoto, S. (2000) High prevalence of antineutrophil cytoplasmic antibody positivity in childhood onset Graves' disease treated with propylthiouracil. J Clin Endocrinol Metab 85, 42704273.

6. Gunton, J.E., Stiel, J., Clifton-Bligh, P., Wilmshurst, E. $\&$ McElduff, A. (2000) Prevalence of positive anti-neutrophil cytoplasmic antibody (ANCA) in patients receiving anti-thyroid medication. Eur J Endocrinol 142, 587.

7. Noh, J.Y., Asari, T., Hamada, N., Makino, F., Ishikawa, N., Abe, Y., Ito, K. \& Ito, K. (2001) Frequency of appearance of myeloperoxidase-antineutrophil cytoplasmic antibody (MPO-ANCA) in Graves' disease patients treated with propylthiouracil and the relationship between MPO-ANCA and clinical manifestations. Clin Endocrinol (oxf) 54, 651-654.

8. Wada, N., Mukai, M., Kohno, M., Notoya, A., Ito, T. \& Yoshioka, N. (2002) Prevalence of serum anti-my- eloperoxidase antineutrophil cytoplasmic antibodies (MPO-ANCA) in patients with Graves' disease treated with propylthiouracil and thiamazole. Endocr $J 49$, 329-334.

9. Bonaci-Nikolic, B., Nikolic, M.M., Andrejevic, S., Zoric, S. \& Bukilica, M. (2005) Antineutrophil cytoplasmic antibody (ANCA)-associated autoimmune diseases induced by antithyroid drugs: comparison with idiopathic ANCA vasculitides. Arthritis Res Ther 7, R1072-1081.

10. Yazisiz, V., Ongut, G., Terzioglu, E. \& Karayalcin, U. (2008) Clinical importance of antineutrophil cytoplasmic antibody positivity during propylthiouracil treatment. Int J Clin Pract, doi: 10.1111/j.1742-1241 .2007.01485.x

11. Cin, M.O., Gursoy, A., Morris, Y., Aydintug, O.T., Kamel, N. \& Gullu, S. (2009) Prevalence and clinical significance of antineutrophil cytoplasmic antibody in Graves' patients treated with propylthiouracil. Int $J$ Clin Pract 63, 299-302.

12. Sera, N., Yokoyama, N., Abe, Y., Ide, A., Usa, T., Tominaga, T., Ejima, E., Kawakami, A., Ashizawa, K. \& Eguchi, K. (2000) Antineutrophil cytoplasmic antibody-associated vasculitis complicating Graves' desease: Report of two adult cases. Acta Med. Nagasaki 45, 33-36.

13. Poomthavorn, P., Mahachoklertwattana, P., TapaneyaOlarn, W., Chuansumrit, A. \& Chunharas, A. (2002) Antineutrophilic cytoplasmic antibody-positive systemic vasculitis associated with propylthiouracil therapy: report of 2 children with Graves' disease. J Med Assoc Thai 85 Suppl 4, S1295-1301.

14. Gao, Y., Chen, M., Ye, H., Guo, X.H., Zhao, M.H. \& Wang, H.Y. (2007) Follow-up of avidity and titre of anti-myeloperoxidase antibodies in sera from pa- 
tients with propylthiouracil-induced vasculitis. Clin Endocrinol (oxf) 66, 543-547.

15. Harper, L., Chin, L., Daykin, J., Allahabadia, A., Heward, J., Gough, S.C., Savage, C.O. \& Franklyn, J.A. (2004) Propylthiouracil and carbimazole associated-antineutrophil cytoplasmic antibodies (ANCA) in patients with Graves' disease. Clin Endocrinol (oxf) 60, 671675.

16. Lam, D.C. \& Lindsay, R.H. (1979) Accumulation of 2-[14C]propylthiouracil in human polymorphonuclear leukocytes. Biochem Pharmacol 28, 2289-2296.

17. Lee, E., Hirouchi, M., Hosokawa, M., Sayo, H., Kohno, M. \& Kariya, K. (1988) Inactivation of peroxidases of rat bone marrow by repeated administration of propylthiouracil is accompanied by a change in the heme structure. Biochem Pharmacol 37, 2151-2153.

18. Williams, J.M., Kamesh, L. \& Savage, C.O. (2005) Translating basic science into patient therapy for
ANCA-associated small vessel vasculitis. Clin Sci (Lond) 108, 101-112.

19. Ye, H., Gao, Y., Guo, X.H. \& Zhao, M.H. (2005) Titre and affinity of propylthiouracil-induced anti-myeloperoxidase antibodies are closely associated with the development of clinical vasculitis. Clin Exp Immunol 142, 116-119.

20. Noh, J.Y., Yasuda, S., Sato, S., Matsumoto, M., Kunii, Y., Noguchi, Y., Mukasa, K., Ito, K., Ito, K., Sugiyama, O., Kobayashi, H., Nihojima, S., Okazaki, M. \& Yokoyama, S. (2009) Clinical characteristics of myeloperoxidase antineutrophil cytoplasmic antibody-associated vasculitis caused by antithyroid drugs. J Clin Endocrinol Metab 94, 2806-2811.

21. Jennette, J.C., Xiao, H. \& Falk, R.J. (2006) Pathogenesis of vascular inflammation by anti-neutrophil cytoplasmic antibodies. J Am Soc Nephrol 17, 1235-1242. 